\title{
Inserción de sonda nasogástrica usando guía esofágica en pacientes con anestesia general en el Hospital Universitario de Neiva
}

\author{
Nasogastric tube insertion using esophageal guide in anesthetized \\ patients at University Hospital in Neiva
}

William Díaz Herrera ${ }^{1 *}$, Héctor Fidel Osorio Zambrano², Miguel Francisco Sandoval Cabrera ${ }^{3}$

\begin{abstract}
Resumen
La inserción de sonda nasogástrica es un procedimiento que consiste en introducir una sonda de polivinilo a través de los orificios nasales, avanzarla por esófago hasta ubicarla en el estómago. En pacientes bajo anestesia general con intubación orotraqueal, la inserción presenta una tasa de fracaso hasta del $50 \%$ según la técnica en el primer intento. Situación que se convierte en un reto hasta para los anestesiólogos experimentados. Objetivo: Evaluar la tasa de éxito y complicaciones en la inserción de una sonda nasogástrica utilizando una guía esofágica comparada con la técnica digital a ciegas. Materiales y métodos: Estudio experimental tipo ensayo clínico controlado de dos grupos de comparación, uno experimental de 19 pacientes (grupo A) en el que se utilizó la guía esofágica para la inserción de la sonda nasogástrica, y el otro de control de 21 pacientes (grupo B) con la técnica digital a ciegas, en un periodo comprendido entre los meses de julio y diciembre del 2012. Resultados: No hubo diferencias significativas entre ambos grupos $(p>0,5)$ en edad, peso y estatura. El tiempo utilizado para inserción de sonda fue significativamente menor en el grupo A con una media en segundos de 70,89 segundos. El número de intentos, en el grupo A fue mucho menor que en el grupo B en un $89.5 \%$ y $57,1 \%$ respectivamente para el $1^{\text {er }}$ y $2^{\text {do }}$ intento, mientras que en el $2^{\text {do }}$ y $3^{\text {er }}$ intento fue de $10.5 \%$ y $42,9 \%$; la incidencia de enrollamiento en el grupo A fue significativamente menor que en el grupo $B$ con $21.1 \%$ y $71,4 \%$ respectivamente. No se presentaron complicaciones en el grupo A con la inserción de la sonda frente a 3 casos en el grupo B, sin presentarse complicaciones severas en ninguno de los dos grupos. Conclusiones: El uso de una guía para el paso de la sonda nasogástrica demostró ser un procedimiento practico y efectivo que permite un procedimiento en menor tiempo cuando se emplea en paciente bajo anestesia general con entubación orotraqueal. El número de intentos para el paso de sonda nasogástrica con guía es mucho menor en comparación al no uso de la misma. Además las deformaciones, que fueron en su mayoría enrollamiento, se presentaron principalmente cuando no se usa guía, y las complicaciones presentadas, el sangrado nasal que solo se presentó en el grupo B.

Por lo tanto, y de acuerdo al análisis estadístico, se recomienda el uso de guía esofágica para el paso de sondas nasogástricas en paciente entubado.

Palabras clave: Intubación Orotraqueal, Anestesia general, Tubo nasogástrico,
\end{abstract}

1. Médico Anestesiólogo, MG. Ecografía perioperatoria. Profesor titular de Anestesiología Universidad Surcolombiana, Hospital Hernando Moncaleano-Perdomo.

2. Médico Anestesiólogo, Hospital Universitario Hernando Moncaleano-Perdomo, Neiva Colombia

3. Residente, Anestesiología y Reanimación, Facultad de Medicina, Universidad Surcolombiana, Neiva Colombia

* Correspondencia: William Díaz Herrera. Correo electrónico: williamdiazherrera@gmail.com

Recepción: 24/03/2016. Revisado: 17/06/2016. Aceptación: 28/11/2016 
R.F.S Revista Facultad de Salud

Julio - Diciembre de 2016;8(2):21-25
Inserción de sonda nasogástrica usando guía esofágica en pacientes con anestesia general en el Hospital Universitario de Neiva

\begin{abstract}
The nasogastric tube insertion is a procedure that consists of introducing a polyvinyl probe through the nostrils, advancing it through the esophagus until it is located in the stomach. In patients under general anesthesia with orotracheal intubation, the insertion has a failure rate of up to $50 \%$ according to the technique in the first attempt. Situation that becomes a challenge even for experienced anesthesiologists. Objective: To evaluate the success rate and complications in the insertion of a nasogastric tube using an esophageal guide compared with blind digital technique. Materials and methods: Experimental study of a controlled clinical trial of two comparison groups, an experimental one of 19 patients (group A) in which the esophageal guide was used for the insertion of the nasogastric tube, and the other control of 21 patients (group B) with digital blind technique, in a period between the months of July and December 2012. Results: There were no significant differences between both groups $(p>0.5)$ in age, weight and height. The time used for probe insertion was significantly lower in group A with a mean in seconds of 70.89 seconds. The number of attempts in group A was much lower than in group B in $89.5 \%$ and $57.1 \%$ respectively for the 1 st and 2 nd attempts, while in the 2 nd and 3 rd attempts it was $10.5 \%$ and $42.9 \%$; the incidence of rolling in group A was significantly lower than in group B with $21.1 \%$ and $71.4 \%$ respectively. There were no complications in group A with the insertion of the probe compared to 3 cases in group B, without presenting severe complications in either of the two groups. Conclusions: The use of a guide for the passage of the nasogastric tube proved to be a practical and effective procedure that allows a procedure in less time when it is used in a patient under general anesthesia with orotracheal intubation. The number of attempts to pass nasogastric tube with guidance is much lower compared to the non-use of it. In addition, the deformations, which were mostly curled, occurred mainly when no guide was used, and the complications presented, nasal bleeding that only occurred in group B.

Therefore, and according to the statistical analysis, the use of an esophageal guide is recommended for the passage of nasogastric tubes in an intubated patient.
\end{abstract}

Keywords: Orotracheal intubation, general anesthesia, nasogastric tube.

\section{Introducción}

La inserción de sondas nasogástricas ( $\mathrm{SNG})$ es un procedimiento común en la práctica médica, se utiliza a partir del siglo XVII con el fin de suministrar nutrición ${ }^{1}$. En la actualidad se ha difundido su empleo en diferentes escenarios como: soporte nutricional enteral, administración de medicamentos, descompresión gástrica ${ }^{2}$ o procedimientos quirúrgicos del tracto digestivo superior, etc. Contextos clínicos que requieren en numerosas ocasiones la participación de un anestesiólogo.

En pacientes bajo anestesia general con intubación orotraqueal y aplicando la técnica de inserción con el cuello en posición neutra, la literatura reporta una tasa de fracaso hasta del $50 \%$ en la inserción de SNG en un primer intento ${ }^{3}$. Situación que se convierte en un reto hasta para los anestesiólogos con experiencia.

Se ha tratado de aplicar diferentes estrategias que faciliten la intervención, entre las que se pueden mencionar el desplazamiento anterior de la laringe, flexionar la cabeza, hacer una presión lateral en el cuello, emplear un dedo enguantado para dirigir la sonda nasogástrica hacia el esófago después de su impactación en la laringe ${ }^{4}$, entre otras. Inclusive algunos autores han propuesto desinflar el manguito del tubo endotraqueal para disminuir la compresión del esófago y facilitar la inserción de la $\mathrm{SNG}^{5}$. Por otro lado, Kayo et al ${ }^{6}$ han planteado el uso de una almohada en pacientes anestesiados, así como el empleo de fórceps y estiletes. Sin embargo, a pesar del esfuerzo por encontrar el mejor método ninguno ha reportado un alto grado de efectividad y confiabilidad.
El presente estudio plantea la posibilidad de utilizar una guía de uso rutinario en procedimientos urológicos endoscópico como guía esofágica para mejorar la eficacia y eficiencia del procedimiento en pacientes bajo anestesia general.

\section{Material y métodos}

Previa aprobación del Comité de Ética Médica del Hospital Universitario Hernando Moncaleano-Perdomo, se llevó a cabo un estudio experimental tipo ensayo clínico controlado en pacientes mayores de 18 años que requerían la inserción de una sonda nasogástrica durante procedimientos quirúrgicos con anestesia general e intubación orotraqueal.

Se realizó la consulta pre-anestésica en los formatos diseñados por la institución para este fin; luego se implementaron los criterios exclusión: pacientes con lesiones en la base del cráneo, inestabilidad en región cervical, tumores de las vías respiratorias superiores, trastornos hemorrágicos, estenosis esofágica, anastomosis del esófago, radioterapia en cabeza o cuello. Al paciente, que si era candidato para el estudio, se le explicó el procedimiento y se firmó consentimiento informado.

En salas de cirugía, primero se midió la longitud de inserción de la sonda, tomando como referencia la distancia hay por la línea media y que une tres puntos: Apófisis xifoides - ala nasal izquierda - lóbulo auricular izquierdo. La técnica de inducción y mantenimiento anestésico dada por el criterio del anestesiólogo tratante. 
Con el paciente anestesiado, se realizó la inserción de la sonda nasogástrica según el grupo seleccionado por la aleatorización previamente estandarizada. Se define como Grupo A: técnica con guía esofágica, y, Grupo B: técnica digital a ciegas.

- Técnica grupo A: Se lubrica tanto la fosa nasal seleccionada con lidocaína jalea como el interior y el exterior de la sonda nasogástrica con lidocaína spray, posteriormente se introduce la GUIA PC ROADRUNNER ${ }^{\circledR}$ con revestimiento hidrofílico licenciado por Cook Medical ${ }^{\circledR}$ : Guía de alma de nitinol recubierta de poliuretano con revestimiento hidrofílico de $145 \mathrm{~cm}$ de longitud x 0,35 $\mathrm{mm}$ de diámetro, de punta recta o angulada y cuerpo flexible empleadas en cirugía endoscópica urológica. Con el paciente en posición cervical neutra se introduce el tubo gástrico con la guía hasta el punto de referencia marcado en el punto tres y finalmente se retira la guía.

- Técnica Grupo B: Se lubrica la fosa nasal seleccionada con lidocaina jalea. Posteriormente, con el paciente en posición cervical neutra, se pasa la sonda con la técnica digital a ciegas, que consiste en introducir dos dedos enguantados en la cavidad oral para dirigir, a manera de pinza, la sonda hacia el esófago cuando se palpe en la laringe.

Se registró el tiempo en segundos (tomado por cronómetro) de la duración del procedimiento hasta la ubicación de la sonda en la cámara gástrica, confirmado por el cirujano y/o la visualización de la salida de contenido gástrico a través del tubo gástrico. Se evaluaron las complicaciones moderadas y severas en el postoperatorio inmediato y en las 24 horas del postoperatorio.

Se establece una base de datos programada en Microsoft office Excel ${ }^{\circ}$, y, posteriormente, se analizaron los datos mediante el Paquete Estadístico SPSS BASIC 15®, diseñado y administrado por la empresa IBM ${ }^{\circledR}$, con licencia gratuita y disponible en su página web.

\section{Resultados}

La muestra estudiada se divide en dos grupos: el primero fue un grupo de intervención con técnica de guía esofágica, con 19 pacientes denominado grupo A, y, el segundo grupo fue el control con la técnica digital a ciegas, con 21 pacientes, denominado grupo $\mathrm{B}$; todos recolectados en forma prospectiva desde 1 de julio a 31 de diciembre 2012, de los cuales no hubo diferencias significativas en edad, peso y estatura, en los dos grupos predominó el género masculino. No se observan diferencias estadísticamente significativas entre ambos grupos $(\mathrm{p}>0,5)$ (tabla 1$)$.

- Tiempo utilizado para inserción de sonda: El tiempo de inserción de la sonda en el grupo A, fue significativamente menor al grupo B, con una media en segundos de 70,89 seg y 214.48 , respectivamente (figura 1 ).
Tabla 1. Características sociodemográficas y clínicas

\begin{tabular}{lcc}
\hline Grupo & A & B \\
\hline Edad: años & $50,74(17,8)$ & $45,6(19,5)$ \\
Genero: M:F & $11: 08$ & $13: 08$ \\
Peso: kg & $66,3(11,3)$ & $65,7(9,7)$ \\
Estatura: cm & $160,7(8,7)$ & $164,2(8.19)$ \\
\hline
\end{tabular}

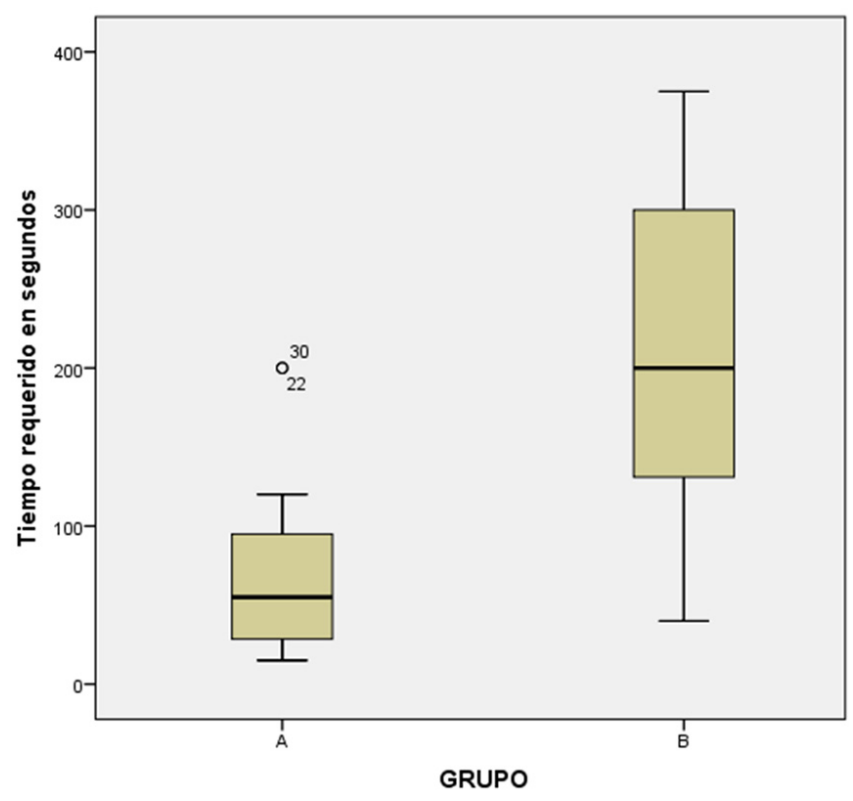

Figura 1. Tiempo utilizado para inserción de sonda.

- Número de intentos en paso de sonda por técnica: El número de intentos en el grupo A fue mucho menor que en el grupo B en un $89.5 \%$ y $57,1 \%$ respectivamente para el $1^{\text {er }}$ y $2^{\text {do }}$ intento, mientras que en el $2^{\text {do }}$ y $3^{\text {er }}$ intento fue de $10.5 \%$ y $42,9 \%$, con un Chi-cuadrado que evidenció asociación, y un valor de $\mathrm{p}<0.05$ que evidenció significación estadística importante (tabla 2).

- Incidencia de enrollamiento y anudación de sonda: En el grupo A, la deformación de la sonda fue significativamente menor que en el grupo B, con $21.1 \%$ y $71,4 \%$ respectivamente con Chi cuadrado de 10,15 demostrando asociación estadística y p 0.001 que evidenció significancia estadística importante (tabla 3 ).

- Incidencia de complicaciones leves y severas: No se presentaron complicaciones en el grupo A con la inserción de la sonda frente al grupo B donde se presentaron 3 casos correspondientes al 14\% (tabla 4). Con P: 0.87 no significativa. No se presentaron complicaciones severas en ninguno de los dos grupos. 
R.F.S Revista Facultad de Salud

Julio - Diciembre de 2016;8(2):21-25
Inserción de sonda nasogástrica usando guía esofágica en pacientes con anestesia general en el Hospital Universitario de Neiva

Tabla 2. Número de intentos en paso de sonda por técnica.

\begin{tabular}{|c|c|c|c|c|c|}
\hline & & & \multicolumn{2}{|c|}{ Número de intentos Total } & \\
\hline & & & 1 y 2 Intentos & 3 y 4 Intentos & \\
\hline & $A$ & Recuento & 17 & 2 & 19 \\
\hline & & $\%$ & $89,5 \%$ & $10,5 \%$ & $100,0 \%$ \\
\hline \multicolumn{6}{|c|}{ TÉCNICA } \\
\hline & B & Recuento & 12 & 9 & 21 \\
\hline & & $\%$ & $57,1 \%$ & $42,9 \%$ & $100,0 \%$ \\
\hline \multirow[t]{2}{*}{ Total } & & Recuento & 29 & 11 & 40 \\
\hline & & $\%$ & $72,5 \%$ & $27,5 \%$ & $100,0 \%$ \\
\hline \multicolumn{3}{|c|}{ Chi-cuadrado de Pearson } & & $5,230^{a}$ & P. 0,022 \\
\hline
\end{tabular}

Tabla 3. Incidencia de enrollamiento y anudación de sonda.

\begin{tabular}{|c|c|c|c|c|}
\hline & & $\begin{array}{l}\text { Deformación } \\
\text { de la sonda }\end{array}$ & $\begin{array}{c}\text { No Deformación } \\
\text { de la sonda }\end{array}$ & Total \\
\hline A & Recuento & 4 & 15 & 19 \\
\hline & $\%$ & $21,1 \%$ & $78,9 \%$ & $100,0 \%$ \\
\hline \multicolumn{5}{|l|}{ TÉCNICA } \\
\hline B & Recuento & 15 & 6 & 21 \\
\hline & $\%$ & $71,4 \%$ & $28,6 \%$ & $100,0 \%$ \\
\hline Chi-cuadrado de Pearson & $10,151^{a}$ & P. 0,001 & & \\
\hline
\end{tabular}

Tabla 4. Incidencia de complicaciones leves.

\begin{tabular}{lcccc}
\hline & \multicolumn{4}{c}{ Complicaciones Inmediatas } \\
\cline { 2 - 4 } & & No complicaciones & $\begin{array}{c}\text { complicaciones } \\
\text { (sangrado nasal) }\end{array}$ & Total \\
\hline Grupo & A & $19(100 \%)$ & $0(0 \%)$ & 19 \\
& B & $18(85,7 \%)$ & $3(14.2 \%)$ & 21 \\
\hline Total & 37 & 3 & 40 \\
\hline
\end{tabular}

\section{Discusión}

El interés por tratar de encontrar una solución práctica y efectiva para el paso de la sonda nasogástrica surge de la observación de la práctica diaria en salas de cirugía del Hospital Hernando Moncaleano-Perdomo, donde se evidenció el alto grado de dificultad que tienen la mayoría de anestesiólogos y residentes en salas de cirugía cuando se requiere el paso de una sonda nasogástrica en pacientes bajo anestesia general.

Según los estudios, en pacientes bajo anestesia general, con intubación orotraqueal y aplicando la técnica de inserción con el cuello en posición neutra, la literatura reporta una tasa de fracaso hasta del $50 \%{ }^{7-9}$ en la inserción al primer intento. Situación que convierte el procedimiento en un reto hasta para los anestesiólogos con experiencia. 
En el presente estudio plantea el procedimiento del paso de sonda nasogástrica con guía, que proporcione un nivel de rigidez mayor con el fin de sobrepasar la resistencia del esófago aumentada por el colapso generado por el manguito del tubo endotraqueal, y se compara con un grupo control, evaluando el tiempo empleado en cada técnica, el número de intentos y sus complicaciones.

Se tomaron 2 poblaciones con características demográficas similares. A un grupo, previa aleatorización, se aplicó el paso de sonda con guía y otro grupo de control, encontrando una significativa reducción del tiempo empleado para el paso de la sonda y un menor rango de variabilidad frente al grupo control que presentó un mayor tiempo empleado con una variabilidad muy amplia, por lo tanto se plantea la posibilidad de que sea puede deber a que el procedimiento es operador dependiente.

En cuanto al número de intentos los resultados, quedó demostrado que con el uso de la guía aumenta la tasa de éxito al primer intento de paso de la sonda en comparación con el grupo control; esto contrasta con los estudios internacionales en los que al aumentar la rigidez de la sonda aumenta el porcentaje de éxito al primer intento. ${ }^{10-14}$.

Las complicaciones leves solo se presentaron en pacientes en grupo control, así como tampoco ocurrieron complicaciones graves o tardías en ningún grupo. Sin embargo, hace falta una muestra mayor de pacientes para evaluar este tipo de complicaciones que resulte en valores estadísticamente significativos que apoyen una conclusión valida es este aspecto.

- Aspectos éticos: El presente estudio se ciñe a las consideraciones estipuladas en las recomendaciones mundiales contenidas en el Informe Belmont de 1978, la Declaración de Helsinki del 2000, y en la resolución No. 2378 de 2008, artículo 2, referente a las buenas prácticas clínicas del Ministerio de Salud De La República de Colombia. Aprobado por del Comité de Ética Médica del Hospital Universitario Hernando Moncaleano-Perdomo de Neiva, mayo 2012.

- Financiación: Recursos propios de los autores.

- Conflicto de intereses: Ninguno declarado.

\section{Referencias}

1. Gharib AM, Stern EJ, Sherbin VL, Rohrmann CA. Nasogastric and feeding tubes. The importance of proper placement. Postgrad Med. 1996 May;99(5):165-8, 174-6.

2. Phillips NM. Nasogastric tubes: an historical context. Medsurg Nurs. 2006 Apr;15(2):84-8.

3. Kirtania J, Ghose T, Garai D, Ray S. Esophageal guidewireassisted nasogastric tube insertion in anesthetized and intubated patients: a prospective randomized controlled study.Anesth Analg. 2012 Feb;114(2):343-8.

4. Mahajan R, Gupta R. Another method to assist nasogastric tube insertion. Can J Anaesth 2005;52:652-3.

5. Kayo R, Kajita I, Cho S, Murakami T, Saito H. A study on insertion of a nasogastric tube in intubated patients. Masui2005; 54:1034-6.

6. Phillips NM, Nasogastric tubes: an historical context. Medsurg Nurs. 2006 Apr;15(2):84-8.

7. Gharib AM, Stern EJ, Sherbin VL, Rohrmann CA. Nasogastric and feeding tubes. The importance of proper placement. Postgrad Med. 1996 May;99(5):165-8, 174-6.

8. Hung $\mathrm{CW}$, Lee WH. A novel method to assist nasogastric tube insertion. Emerg Med J. 2008 Jan;25(1):23-5.

9. Spurrier EJ, Johnston AM. Use of nasogastric tubes in trauma patients - a review. J R Army Med Corps. 2008 Mar;154(1):10-3.

10. Fuchs J, Schummer C, Giesser J, Bayer O, Schummer W. Detection of tracheal malpositioning of nasogastric tubes using endotracheal cuff pressure measurement. Acta Anaesthesiol Scand. 2007 Oct;51(9):1245-9.

11. Hung CW, Lee WH. A novel method to assist nasogastric tube insertion. Emerg Med J. 2008; Jan;25(1):23-5.

12. Chun DH, Kim NY, Shin YS, Kim SH. A randomized, clinical trial of frozen versus standard nasogastric tube placement. World J Surg. 2009 Sep;33(9):1789-92.

13. Appukutty J, Shroff PP. Nasogastric tube insertion using different techniques in anesthetized patients: a prospective, randomized study. Anesth Analg. 2009 Sep; 109(3):832-5. 